\title{
Light-Matter Coupling Conference (PLMCN5) to be Held in Glasgow in June 2005
}

The 5th International Conference on Physics of Light-Matter Coupling in Nanostructures (PLMCN5) will be held June 8-11, 2005, in Glasgow, Scotland. The conference is focused on the fundamental and technological issues relevant to the realization of a new generation of optoelectronic devices based on advanced low-dimensional and photonic structures such as low-threshold polariton lasers, new optical switches, single-photon emitters, and photonic-bandgap structures. It will bring together scientists from academia and industry who are active in the fields of semiconductor optics and materials science to review recent achievements in the fundamental understanding of strong light-matter coupling, as well as to follow progress in the development of epitaxial and processing technologies of http://plmcn5.phys.strath.ac.uk/

wide-bandgap semiconductor and organic nanostructures and microcavities that are providing the basis for advanced optical studies.

The scope of the conference covers physics and applications of a variety of phenomena related to light-matter coupling in solids. Topics to be discussed include

- microcavity physics and devices;

- photonic crystals;

- the physics and application of quantum dots;

- basic exciton-polariton physics;

- spin-related optical phenomena in microcavities;

- the growth and characterization of advanced wide-bandgap nanostructures (e.g., GaN, $\mathrm{ZnSe}, \mathrm{ZnO}$, and organic materials); and

- novel device concepts (e.g., polariton lasers, single-photon emitters, and optical switches).

Invited speakers include J. Wierer (Lumileds) on "State-of-the-Art III-N LEDs: Microcavity and Photonic Crystal Effects," E. Kapon (EPFL, Lausanne) on "Ordered Semiconductor Quantum Dots Made by Patterned Epitaxial Growth," and T. Tawara (NTT, Japan) on "InGaN Cavity Polaritons."

The conference is chaired by Robert Martin (Strathclyde University), Kevin O'Donnell (Strathclyde University), and Alexey Kavokin (Clermont Ferrand). For further information, e-mail plmcn5@ phys.strath.ac.uk or access Web site http://plmcn5.phys.strath.ac.uk/. 